\title{
Effect of Citrate-Acidified Dialysate on Intact Parathyroid Hormone in Prevalent Hemodialysis Patients: A Matched Retrospective Cohort Study
}

\author{
Linda H Ficociello $\mathbb{D}^{\prime}$ \\ Meijiao Zhou' \\ Claudy Mullon $\mathbb{D}^{1,2}$ \\ Michael S Anger (1D ${ }^{1,2}$ \\ Robert J Kossmann'
}

'Fresenius Medical Care, Global Medical Office, Waltham, MA, USA; ${ }^{2}$ Fresenius Medical Care, Renal Therapies Group, Waltham, MA, USA
Correspondence: Robert J Kossmann Email Robert.Kossmann@fmc-na.com
Background: It has been proposed that substituting citrate-acidified dialysate (CAD) solutions for acetate-acidified dialysate (AAD) could improve hemodynamics and dialysis tolerance and reduce the requirement for systemic anticoagulation. Citrate chelates ionized calcium, but long-term effects of CAD use during maintenance hemodialysis have not been well studied. While many studies of the effects of CAD on serum calcium and intact parathyroid hormone (iPTH) have been short-term or have been limited by sample size, we aimed to determine if there are any long-term (i.e., 6-month) changes from pre-dialysis iPTH levels when patients are switched from AAD to CAD.

Methods: This retrospective cohort study compared various clinical parameters, including pre-dialysis $\mathrm{iPTH}$ and serum calcium as well as single pool $\mathrm{Kt} / \mathrm{V}$, from eligible patients who received in-center hemodialysis thrice-weekly in geographically matched CAD $(n=3)$ or AAD clinics $(n=12)$. CAD clinics were defined as clinics converting from AAD to CAD if $>85 \%$ of the patients were prescribed CAD after implementation of CAD within the clinic. Results: Pre-dialysis iPTH was not significantly different from baseline to 6-month followup within either $\mathrm{CAD}$ or AAD clinics. Moreover, the mean change from baseline to month 6 in iPTH between patients $(\mathrm{n}=142)$ in CAD clinics $(-17 \mathrm{pg} / \mathrm{mL})$ and patients $(\mathrm{n}=671)$ in AAD clinics $(13 \mathrm{pg} / \mathrm{mL})$ was similar $(p=0.24)$. Likewise, the differences in the mean change in serum calcium concentrations and dialysis adequacy (single pool $\mathrm{Kt} / \mathrm{V}$ ) were not significant between $\mathrm{CAD}$ and $\mathrm{AAD}$ clinics. For subgroups of patients who were never prescribed cinacalcet or calcium-based phosphate binders, there were no significantly different categorical shifts in $\mathrm{PPTH}$ between $\mathrm{CAD}$ and AAD clinics.

Conclusion: Similar trends in single pool $\mathrm{Kt} / \mathrm{V}$, iPTH, and serum calcium levels were observed in clinics that switched from AAD to CAD versus the geographically matched AAD clinics. These results support CAD as a potential alternative to AAD in hemodialysis. Keywords: hemodialysis, citrate-acidified dialysate, acetate-acidified dialysate, parathyroid hormone, iPTH, serum calcium

\section{Introduction}

For hemodialysis, the $\mathrm{pH}$ of the dialysate is lowered using an acidifier, typically a low concentration of acetic acid, in order to prevent precipitation by contact between bicarbonate and calcium and magnesium divalent cations. ${ }^{1}$ However, acetic acid in dialysate has been associated with vasodilation, hypotension, and vascular calcification. ${ }^{1,2}$ Citric acid has been introduced as an alternative acidifier for dialysate in bicarbonate dialysis. Citrate-acidified dialysate (CAD) allows for dialysis to occur without the use of acetate or with a reduced concentration of 
acetate $(0.3 \mathrm{mEq} / \mathrm{L})$. Citrate is a known chelator of calcium that binds to ionized calcium, resulting in a lower serum concentration of ionized calcium available for participation in the clotting cascade. ${ }^{3,4}$

Parathyroid hormone (PTH) and calcium are regulators in a feedback loop involving the bones, kidneys, and the parathyroid glands. ${ }^{5}$ PTH regulates the body's calcium/ phosphate balance through its actions on bone and kidney. In turn, serum calcium levels regulate secretion of PTH, stimulating PTH production when serum calcium concentrations are low. ${ }^{6,7}$ As such, hypocalcemia can contribute to secondary hyperparathyroidism. Hyperphosphatemia and decreased renal production of 1,25-dihydroxyvitamin D3 also contribute to secondary hyperparathyroidism, which may have to be managed in patients with chronic kidney disease (CKD) ${ }^{8,9}$ Traditionally, therapeutic approaches include the administration of active vitamin D and phosphate binders to manage secondary hyperparathyroidism. Additionally, if treatment induces hypercalcemia and hyperphosphatemia, use of a calcimimetic may be a therapeutic option to regain balance of these regulators. ${ }^{9-12}$

It has been proposed that substituting CAD solutions for acetate-acidified dialysate (AAD) in bicarbonate dialysate could improve hemodynamic and dialysis tolerance, enhance biocompatibility with reduced extracorporeal thrombosis risk, and reduce the requirement for anticoagulation, which can be particularly important for patients at risk of bleeding. ${ }^{13} \mathrm{CAD}$ may also improve dialysis adequacy, presumably due to the non-systemic anticoagulant effects on the dialyzer. ${ }^{14-16}$ A multicenter, prospective analysis of patients on hemodialysis treated with CAD demonstrated that $\mathrm{CAD}$ could reduce heparin doses while maintaining dialysis adequacy. ${ }^{17}$ In this study, mean total calcium and ionized calcium decreased during dialysis using CAD; however, values of mean post-dialysis total calcium and post-dialysis ionized calcium were within the physiologic range. While this study did not evaluate the effect of CAD on intact PTH (iPTH), other studies have not found a significant change in $\mathrm{PTH}$ as a result of using CAD. Grundström et al found no significant intra-dialytic shift in PTH levels with CAD, while AAD resulted in a significant decrease in post-dialysis PTH levels. ${ }^{18}$ Similarly, Šafránek et al found that AAD was associated with a substantial decrease in $\mathrm{PTTH}$, while CAD resulted in a non-significant increase. ${ }^{19}$ This study also demonstrated that calcium levels increased during hemodialysis with both $\mathrm{CAD}$ and $\mathrm{AAD}$, but the increases were significantly smaller with CAD. In addition, Kossmann et al found a small increase in pre-hemodialysis $\mathrm{IPTH}$ during the first 3 months of using CAD dialysate. ${ }^{20}$ In contrast, Park et al found that relative to AAD, CAD resulted in decreased iPTH and reduced heparin requirements. ${ }^{21}$

Because previous studies on outcomes related to CAD versus $A A D$ have been limited by sample size and study length or have had potential confounders due to changes in the management of chronic kidney disease-mineral bone disorder, we aimed to determine if there are any long-term changes in pre-dialysis levels of iPTH when patients are switched from AAD to CAD.

\section{Materials and Methods Study Design}

This retrospective, geographically matched cohort study used de-identified data extracted from the Fresenius Kidney Care clinical data warehouse. Clinics included in the study were identified as either CAD clinics (clinics converting from $\mathrm{AAD}$ to $\mathrm{CAD}[>85 \%$ of the patients prescribed $\mathrm{CAD}$ after implementation of $\mathrm{CAD}$ within the clinic] between 1/1/2009 and 12/31/2011) or AAD clinics (clinics with continued reliance on AAD). These dates were selected to exclude periods of time (eg, 2013) when other, non-dialysate-related changes to the management of mineral bone disease were implemented across large dialysis organizations. ${ }^{22}$ Inclusion of such time periods into the study would have introduced additional confounders to our analyses due to changing targets and therapeutic interventions. In CAD clinics, Citrasate ${ }^{\circledR}$ (Fresenius Medical Care NA, Waltham, Mass, USA), containing $2.4 \mathrm{mEq}$ of citric acid (citrate) and dialysate calcium concentration of $2.5 \mathrm{mEq}$ per liter, ${ }^{17}$ was used. When AAD was used, calcium concentrations could be 2.0, 2.2, 2.5, 3.0, or 3.5 $\mathrm{mEq} / \mathrm{L}$.

Three clinics implemented use of CAD between 2009 and 2011 and were matched in a 1:4 ratio to geographically matched AAD facilities. AAD facilities also were matched to the CAD facilities based on a similar number of in-center patients on hemodialysis who met the inclusion/exclusion criteria of the study during the 30-day inclusion period. Patients receiving home dialysis and patients receiving in-center nocturnal dialysis were excluded from the study. For patients at CAD clinics, the start date of the patient's earliest CAD order was defined as the CAD patient index date. The index date for patients 
in AAD clinics was set to 15 days after the earliest CAD start date in the matched CAD facility.

Eligible in-center hemodialysis patients received dialysis in the selected CAD or AAD clinics. For inclusion in the analysis, patients had to be in-center hemodialysis patients at a Fresenius Kidney Care facility and aged 18 to 89 years on the index date. These patients continuously received hemodialysis at the facility for at least 6 months prior to the CAD conversion/index and continued to receive treatment for at least an additional 6 months. All patients included had available pre-dialysis iPTH values.

The observation period was defined as baseline (6 months before the index date) and 6-month follow-up (6 months after the index date). During the observation period, iPTH was assessed quarterly, serum calcium was measured monthly, and prescribed dialysate calcium treatments were recorded. For the purposes of this analysis, medication must have been prescribed for at least 3 months at baseline or follow-up to be considered "prescribed" during that period.

Serum calcium and iPTH were compared at baseline and follow-up. Data on the use of calcium-based phosphate binder, oral cinacalcet, and intravenous (IV) vitamin $\mathrm{D}$ were extracted and calculated for baseline and followup. This study was determined to be exempt by the New England Independent Review Board due to the use of deidentified retrospective data with no impact on care of patients.

\section{Statistical Analyses}

Demographics were presented as the mean (standard deviation $[\mathrm{SD}]$ ) for continuous variables and the number of patients (\%) for categorical variables. Comparisons of demographics between $\mathrm{CAD}$ and AAD clinics were performed using a 2-sample $t$-test for continuous variables and chi-square test for categorical variables. The values of all clinical variables were averaged over baseline and follow-up for each patient. Comparisons of data between baseline and follow-up within the clinic were made using paired $t$-tests and McNemar's tests for continuous and categorical variables. Comparisons between $\mathrm{CAD}$ and AAD clinics were made using a 2-sample $t$-test for continuous variables and logistic regression with repeated measures for categorical variables. Two-sided $p$-values $<0.05$ were considered statistically significant.

To reduce the potential confounding effect of mineral and bone disorder medications on iPTH, pre-defined subgroup analyses were performed for patients without a prescription for calcium-based phosphate binders, patients without a prescription for cinacalcet, and patients without a modification of vitamin D usage. Similarly, the impact of dialysate on serum phosphorus levels was also analyzed in the subgroup of patients not prescribed phosphate binders. Because the calcium concentration in AAD can vary, sensitivity analyses were performed on the subset of patients treated with dialysate containing $2.5 \mathrm{mEq} / \mathrm{L}$ of calcium at both baseline and follow-up. All analyses were carried out with SAS software version 9.4 (SAS Institute Inc, Cary, NC, USA).

\section{Results}

Of the 815 patients across the 15 sites who met eligibility criteria, 813 patients were included in this analysis (2 patients were excluded as a result of missing $\mathrm{PPTH}$ data): 142 in the CAD group and 671 in the AAD group. Baseline patient demographics were similar, but some statistically significant differences were noted between the CAD and AAD clinics overall. The average (SD) age was 65.3 (13.9) years and 62.6 (14.4) years in the CAD and AAD clinics, respectively. There were proportionally more White patients at CAD clinics (87.3\%) than at AAD clinics (78.5\%), and more Hispanic patients at CAD clinics (47.2\%) than at AAD clinics (36.7\%) (Table 1). Patients at CAD clinics had higher scores on the Charlson Comorbidity Index (3.0 versus 2.7 ) and were also more likely to have congestive heart failure compared with those at AAD clinics (26.0\% versus $16.3 \%)$. A complete summary of baseline characteristics can be found in Table 1.

Pre-dialysis iPTH was not significantly different from baseline to follow-up at either type of clinic; at CAD clinics, the mean iPTH decreased by $17 \mathrm{pg} / \mathrm{mL}(4.1 \%$ reduction, $p=0.49$ ), and at AAD clinics, mean iPTH increased by $13 \mathrm{pg} / \mathrm{mL}$ (3.8\% increase, $p=0.13)$. The longitudinal change in iPTH was similar between CAD and AAD clinics $(p=0.24)$. The change in mean serum calcium from baseline to follow-up was not significant in CAD clinics. Although a small increase in mean (SD) serum calcium of $0.05(0.4) \mathrm{mg} / \mathrm{dL}(p=0.007)$ was observed in AAD clinics, the difference between mean change in serum calcium between CAD and AAD clinics was not significant. In a sensitivity analysis, these patterns remained when data from 14 patients who switched from $\mathrm{CAD}$ to $\mathrm{AAD}$ during the follow-up period were removed from the CAD data set (Supplementary Table 1). 
Table I Patient Demographics and Treatment Parameters Among Clinics Using CAD and AAD

\begin{tabular}{|c|c|c|c|}
\hline \multirow[t]{2}{*}{ Characteristics } & \multicolumn{2}{|c|}{ All Patients } & \multirow[t]{2}{*}{ P-Value } \\
\hline & CAD $(n=\mid 42)$ & AAD $(n=67 I)$ & \\
\hline Age, years (SD) & $65.3(13.9)$ & $62.6(14.4)$ & 0.04 \\
\hline Height, cm (SD) & $164.9(12.1)$ & $165.8(11.3)$ & 0.38 \\
\hline Dialysis vintage, years (SD) & $3.9(2.8)$ & $4.4(3.7)$ & 0.08 \\
\hline $\mathrm{CCl}^{*}(\mathrm{SD})$ & $3.0(1.1)$ & $2.7(0.9)$ & 0.009 \\
\hline \multicolumn{3}{|l|}{ Race, n (\%) } & 0.04 \\
\hline White & $124(87.3)$ & $527(78.5)$ & \\
\hline Black & $6(4.2)$ & $65(9.7)$ & \\
\hline Other & $12(8.5)$ & $79(11.8)$ & \\
\hline \multicolumn{3}{|l|}{ Ethnicity, n (\%) } & 0.02 \\
\hline Hispanic & $67(47.2)$ & $246(36.7)$ & \\
\hline Not Hispanic & $75(52.8)$ & $425(63.3)$ & \\
\hline \multicolumn{3}{|l|}{ Sex, n (\%) } & 0.20 \\
\hline Female & $73(5 \mathrm{I} .4)$ & $305(45.5)$ & \\
\hline Male & $69(48.6)$ & $366(54.5)$ & \\
\hline \multicolumn{3}{|l|}{ Vascular access, n (\%) } & $0.4 I^{\dagger}$ \\
\hline Fistula & $90(63.4)$ & 476 (70.9) & \\
\hline Graft & $13(9.2)$ & $66(9.8)$ & \\
\hline Catheter & $28(19.7)$ & $117(17.4)$ & \\
\hline Mixed & $5(3.5)$ & $12(1.8)$ & \\
\hline Unknown & $6(4.2)$ & 0 & \\
\hline \multicolumn{3}{|l|}{ Access with catheter, $\mathrm{n}(\%)$} & 0.52 \\
\hline Yes & $28(19.7)$ & $117(17.4)$ & \\
\hline No & $114(80.3)$ & $554(82.6)$ & \\
\hline \multicolumn{3}{|c|}{ End-stage kidney disease causes, n (\%) } & $0.10^{\ddagger}$ \\
\hline Diabetes & $76(53.5)$ & $349(52.0)$ & \\
\hline Hypertension & $21(14.8)$ & $149(22.2)$ & \\
\hline Glomerulonephritis & $10(7.0)$ & $60(8.9)$ & \\
\hline Polycystic kidney disease & $\mathrm{I}(0.7)$ & $23(3.4)$ & \\
\hline Other & $34(23.9)$ & $90(13.4)$ & \\
\hline \multicolumn{4}{|l|}{ Comorbid conditions, n (\%) } \\
\hline Diabetes mellitus, $\mathrm{n}(\%)$ & $77(58.8)$ & $330(53.4)$ & 0.26 \\
\hline Congestive heart failure, $\mathrm{n}(\%)$ & $34(26.0)$ & $101(16.3)$ & 0.009 \\
\hline
\end{tabular}

Notes: *Sample size for $\mathrm{CCl}$ was $\mathrm{n}=|3|$ in $\mathrm{CAD}$ and $\mathrm{n}=618$ in AAD. ${ }^{\dagger}$ The category of unknown was treated as missing in the $p$-value calculation. ${ }^{\ddagger}$ We collapsed the categories of glomerulonephritis, polycystic kidney disease, and other as one group in the $p$-value calculation.

Abbreviations: AAD, acetate-acidified dialysate; CAD, citrate-acidified dialysate; CCI, Charlson Comorbidity Index; SD, standard deviation. 
Mean baseline serum phosphorus levels were higher in CAD clinics than AAD clinics $(5.55 \mathrm{mg} / \mathrm{dL}$ versus $5.46 \mathrm{mg} / \mathrm{dL}$ ) and decreased to $5.44 \mathrm{mg} / \mathrm{dL}$ in both groups at follow-up. The changes in serum phosphorus were not statistically significant in either clinic group or between clinic groups (Table 2). Single pool Kt/V (SD) increased in both CAD (0.06 [0.19], $p=0.0004)$ and AAD clinics (0.03 [0.23], $p=0.001)$ from baseline to follow-up. The difference between clinics was not statistically significant.

Mean (SD) prescribed dialysate calcium increased from baseline to follow-up in CAD clinics (2.3 [0.1] $\mathrm{mEq} / \mathrm{L}$ to $2.5[0.1] \mathrm{mEq} / \mathrm{L}, p<0.0001)$, but it did not increase in AAD clinics $(2.4[0.2] \mathrm{mEq} / \mathrm{L}$ at both baseline and follow-up). The difference between mean change in prescribed dialysate calcium between $\mathrm{CAD}$ and $\mathrm{AAD}$ clinics was small, but statistically significant $(p<$ $0.0001)$. Use of cinacalcet increased significantly from baseline to follow-up by $5.5 \%$ at AAD clinics ( $p<$ 0.0001 ) but did not change at CAD clinics. Use of calcium-based phosphate binders increased significantly at both clinics, but to a lesser degree at CAD clinics $(18.6 \%$ at $\mathrm{AAD}$ clinics versus $7.0 \%$ at CAD clinics, $p=$ 0.01). The difference in mean change for both cinacalcet and calcium-based phosphate binders between CAD and AAD clinics was significant ( $p=0.01$ for each). From baseline to follow-up, use of IV vitamin D significantly increased in both clinic types; however, there was no significant difference in the change in IV vitamin $\mathrm{D}$ between clinics.

Figure 1 illustrates the change in the number of patients in $\mathrm{PTH}$ categories among CAD and AAD clinics for all patients. This analysis showed no statistically significant categorical shifts for those in CAD clinics; however, there were statistically significant changes for those in the AAD clinics, as there was a marked increase in the number of patients with iPTH $>300 \mathrm{pg} / \mathrm{mL}$. Although a difference was seen within the AAD group, there was no statistically significant difference between the CAD and AAD groups.

Of the 142 patients in the CAD group, 42 received dialysate treatment with AAD containing $2.5 \mathrm{mEq} / \mathrm{L}$ during the baseline period and CAD containing $2.5 \mathrm{mEq} / \mathrm{L}$ of calcium during the follow-up period. Similarly, 400 patients in the AAD group received dialysate treatment with AAD containing $2.5 \mathrm{mEq} / \mathrm{L}$ during the baseline and follow-up periods. During follow-up, both subgroups of patients demonstrated significant increases in $\mathrm{PTTH}$ from baseline, but there were no differences between the CAD and AAD clinics (Supplementary Table 2). In the CAD subgroup, patients experienced a mean (SD) reduction in serum calcium of $0.19(0.3) \mathrm{mg} / \mathrm{dL}$ during follow-up $(p=$ 0.0006). Serum calcium levels remained constant in those patients receiving AAD with $2.5 \mathrm{mEq} / \mathrm{L}$ during baseline and follow-up. There were 41 patients in the CAD group who were prescribed dialysate with $2.2 \mathrm{mEq} / \mathrm{L}$ during baseline and then CAD with $2.5 \mathrm{mEq} / \mathrm{L}$ during the followup period. These patients did not experience significant changes in iPTH or serum calcium concentrations (Supplementary Table 3).

Cinacalcet was prescribed for $27 \%$ of the patients from CAD clinics and $20 \%$ from AAD clinics during the study. Results were similar when patients receiving cinacalcet were excluded from the analysis (Figure 2). Similarly, calcium-based phosphate binders were prescribed to $30 \%$ of the patients from CAD clinics and 53\% from AAD clinics. When these patients were excluded from the analysis, categorical iPTH analysis did not demonstrate significant differences within or between clinic types (Figure 3).

Because more than $80 \%$ of the patients were receiving IV vitamin $\mathrm{D}$ at baseline, we conducted a subgroup analysis on those patients who did not have their vitamin $\mathrm{D}$ usage modified during the observation period (CAD: $\mathrm{n}=129$; AAD: $\mathrm{n}=594)$. Among this subgroup, no significant differences were observed between the 2 clinic groups $(p=0.20)$. Patients in CAD clinics exhibited nonsignificant decreases in iPTH (mean [SD]: 29 [285] pg/ $\mathrm{mL}$ ) and patients in AAD clinics exhibited non-significant increases in iPTH (mean [SD]: 6 [219] pg/mL).

Among patients without a prescription for phosphate binders (CAD: $n=80$; AAD: $n=83$ ) mean (SD) baseline serum phosphorus levels were greater in the CAD clinics (5.85 [1.48] $\mathrm{mg} / \mathrm{dL}$ ) than in AAD clinics (4.95 [1.34] mg/dL). In this subgroup, no significant changes in serum phosphorus were observed in either clinic group ( $p=0.12$ for CAD and $p=0.72$ for $\mathrm{AAD})$ or between clinic groups $(p=0.17$ ).

\section{Discussion}

Results from our analyses demonstrated that over a 6-month period, there was no significant increase in predialysis $\mathrm{PPTH}$ among patients in CAD clinics or those in AAD clinics. Pre-dialysis iPTH was numerically decreased in CAD clinics, but this difference was not considered clinically significant. Additionally, the change in iPTH was not different between clinic types. Our results are consistent with results from data previously published 
Table 2 Changes in iPTH, Serum Calcium, and CKD-MBD Medications: CAD ( $n=142)$ and AAD ( $n=671)$ Patients

\begin{tabular}{|c|c|c|c|c|c|}
\hline & Baseline (BL) & Follow-Up (FU) & $\Delta$ BL vs FU & $P$-value (BL vs FU) & $P$-value (CAD vs AAD) \\
\hline \multicolumn{6}{|c|}{ iPTH, pg/mL (SD) } \\
\hline CAD & $4 I I(46 I)$ & $394(394)$ & $-17(287)$ & 0.49 & \multirow[t]{2}{*}{0.24} \\
\hline AAD & $338(266)$ & $351(268)$ & $13(229)$ & 0.13 & \\
\hline \multicolumn{6}{|c|}{ Serum calcium, mg/dL (SD) } \\
\hline CAD & $8.9(0.7)$ & $9.0(0.8)$ & $0.07(0.6)$ & 0.14 & \multirow[t]{2}{*}{0.61} \\
\hline AAD & $9.0(0.6)$ & $9.1(0.6)$ & $0.05(0.4)$ & 0.007 & \\
\hline \multicolumn{6}{|c|}{ Serum phosphorus, mg/dL (SD) } \\
\hline CAD & $5.55(1.39)$ & $5.44(1.26)$ & $-0.11(0.78)$ & 0.10 & \multirow[t]{2}{*}{0.31} \\
\hline AAD & $5.46(1.31)$ & $5.44(1.32)$ & $-0.03(0.88)$ & 0.46 & \\
\hline \multicolumn{6}{|c|}{ Prescribed dialysate calcium, mEq/L (SD) } \\
\hline CAD & $2.3(0.1)$ & $2.5(0.1)$ & $0.19(0.1)$ & $<0.0001$ & \multirow[t]{2}{*}{$<0.0001$} \\
\hline AAD & $2.4(0.2)$ & $2.4(0.2)$ & $0.008(0.1)$ & 0.08 & \\
\hline \multicolumn{6}{|c|}{ Prescribed HD treatment time, minutes (SD) } \\
\hline CAD & $228(27)$ & $229(28)$ & I.I (7.7) & 0.10 & \multirow[t]{2}{*}{0.49} \\
\hline AAD & $230(24)$ & $231(24)$ & $0.6(7.3)$ & 0.03 & \\
\hline \multicolumn{6}{|c|}{ Pre-HD weight, kg (SD) } \\
\hline CAD & $79.4(19.2)$ & $79.0(19.2)$ & $-0.50(2.72)$ & 0.03 & \multirow[t]{2}{*}{0.23} \\
\hline AAD & $80.8(22.9)$ & $80.7(22.8)$ & $-0.17(2.96)$ & 0.13 & \\
\hline \multicolumn{6}{|c|}{ Single pool Kt/V (SD) } \\
\hline CAD & $1.73(0.25)$ & $1.79(0.29)$ & $0.06(0.19)$ & 0.0004 & \multirow[t]{2}{*}{0.11} \\
\hline AAD & $1.74(0.30)$ & $1.77(0.28)$ & $0.03(0.23)$ & 0.001 & \\
\hline \multicolumn{6}{|c|}{ Calcium-based phosphate binder, \% } \\
\hline CAD & $20.4 \%(29 / 142)$ & $27.5 \%(39 / 142)$ & $7.0 \%(10 / 142)$ & 0.004 & \multirow[t]{2}{*}{0.01} \\
\hline AAD & $27.7 \%(\mid 86 / 67 I)$ & $46.4 \%(3 I I / 67 I)$ & $18.6 \%(|25 / 67|)$ & $<0.0001$ & \\
\hline \multicolumn{6}{|c|}{ Cinacalcet, \% } \\
\hline CAD & $17.6 \%(25 / 142)$ & $17.6 \%(25 / 142)$ & 0 & 1.00 & \multirow[t]{2}{*}{0.01} \\
\hline$A A D$ & II.0\% (74/67I) & $16.5 \%(|1| / 67 \mid)$ & $5.5 \%(37 / 67 I)$ & $<0.0001$ & \\
\hline \multicolumn{6}{|c|}{ IV vitamin D, \% } \\
\hline CAD & $86.6 \%(123 / 142)$ & $93.0 \%(132 / 142)$ & $6.3 \%(9 / 142)$ & 0.01 & \multirow[t]{2}{*}{0.26} \\
\hline$A A D$ & $83.0 \%(557 / 67 I)$ & $87.6 \%(588 / 67 I)$ & $4.6 \%(3 I / 67 I)$ & 0.0004 & \\
\hline
\end{tabular}

Abbreviations: AAD, acetate-acidified dialysate; CAD, citrate-acidified dialysate; CKD, chronic kidney disease; HD, hemodialysis; iPTH, intact parathyroid hormone; IV, intravenous; MBD, mineral bone disorder; SD, standard deviation.

by Grundström et al in which pre-dialysis levels of PTH calcium). ${ }^{18}$ Our results were consistent with these prior did not vary between CAD $(3 \mathrm{mEq} / \mathrm{L}$ citrate and $3 \mathrm{mEq} / \mathrm{L}$ data despite the use of different concentrations of citrate in calcium) and $\mathrm{AAD}(3 \mathrm{mEq} / \mathrm{L}$ acetate and $3 \mathrm{mEq} / \mathrm{L}$ the dialysate $(2.4 \mathrm{mEq} / \mathrm{L}$ in the present study). 
CAD Patients ( $n=142)$

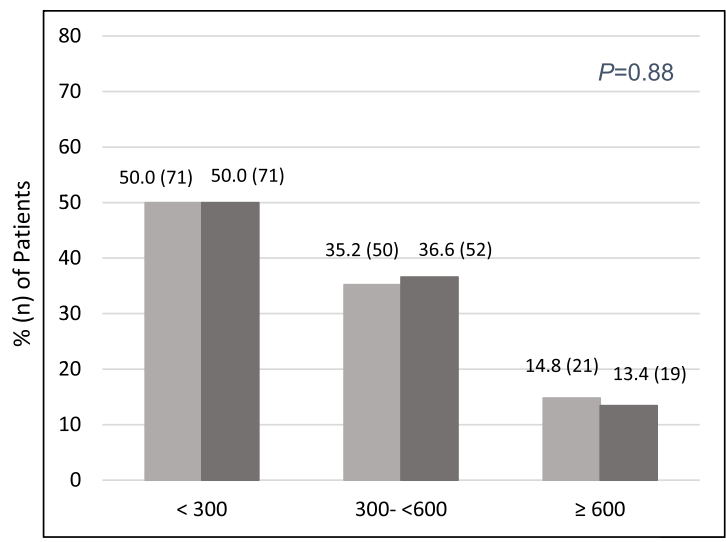

iPTH levels (pg/ml)
AAD Patients $(n=671)$

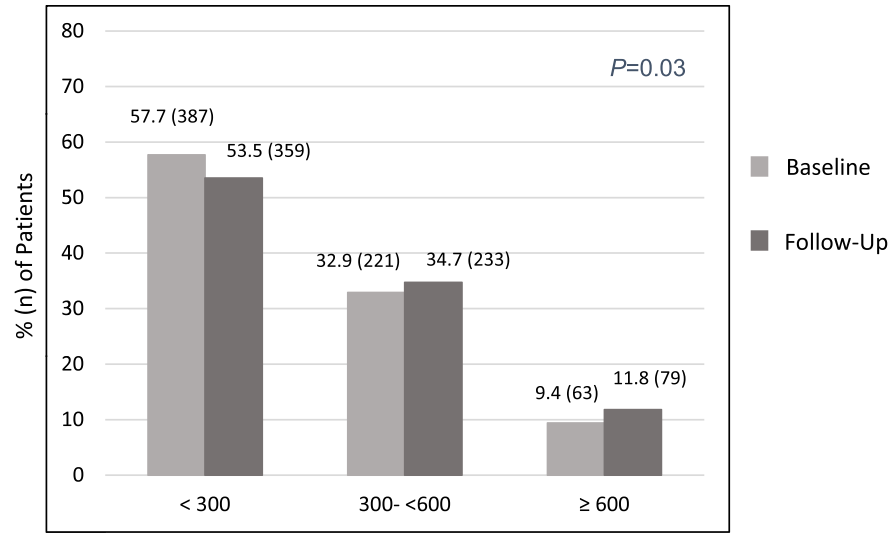

$\mathrm{P}=0.27$

(CAD vs. AAD)

iPTH levels (pg/ml)

Figure I Number of individuals in each iPTH category at baseline and 6 months: overall population.

Abbreviations: AAD, acetate-acidified dialysate; CAD, citrate-acidified dialysate; $i \mathrm{PTH}$, intact parathyroid hormone.

CAD Patients $(n=104)$

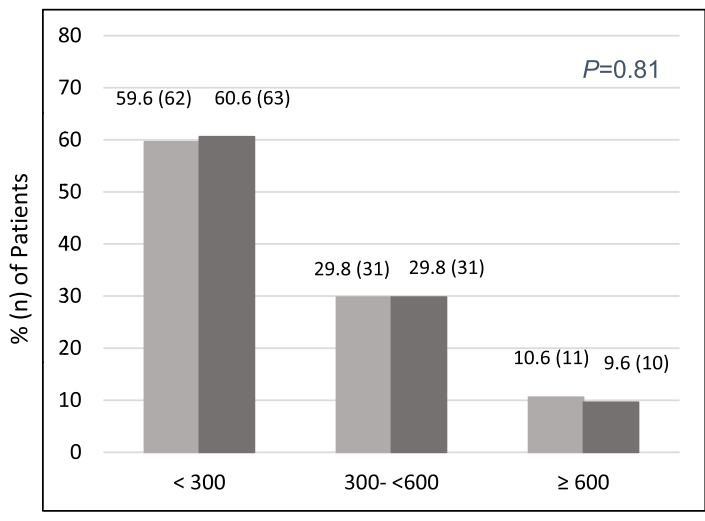

iPTH levels (pg/ml)
AAD Patients $(n=534)$

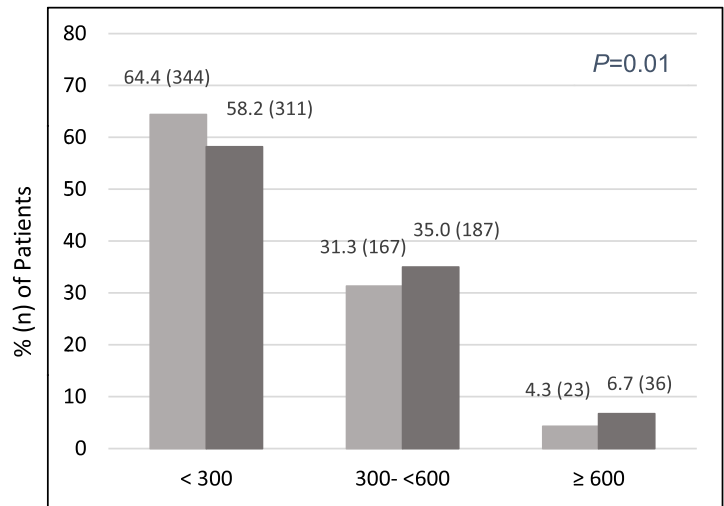

iPTH levels $(\mathrm{pg} / \mathrm{ml})$
Baseline

Follow-Up

Figure 2 Individuals in each iPTH category at baseline and 6 months: patients not taking cinacalcet.

Abbreviations: $A A D$, acetate-acidified dialysate; CAD, citrate-acidified dialysate; iPTH, intact parathyroid hormone.

A recent retrospective study from South Korea evaluated 75 patients who underwent hemodialysis with AAD $(8.0 \mathrm{mEq} / \mathrm{L}$ acetate, $2.75 \mathrm{mEq} / \mathrm{L}$ calcium) for 6 months and were then switched to hemodialysis with CAD (2.0 $\mathrm{mEq} / \mathrm{L}$ citrate, $2.5 \mathrm{mEq} / \mathrm{L}$ calcium) for an additional 6 months. ${ }^{21}$ The authors found that $\mathrm{iPTH}$ increased over the $\mathrm{AAD}$ period and then decreased over the $\mathrm{CAD}$ period (AAD: $73 \mathrm{pg} / \mathrm{mL}$ versus CAD: $-107 \mathrm{pg} / \mathrm{mL}, p<0.001$ ). Serum calcium decreased during the AAD period but increased during the CAD period (AAD: $-0.17 \mathrm{mg} / \mathrm{dL}$ versus CAD: $0.23 \mathrm{mg} / \mathrm{dL}, p=0.006)$. The authors of this study concluded that the relationship between $\mathrm{PPTH}$ and citrate was difficult to determine because calcium-based phosphate binders were prescribed at different rates during the CAD and AAD periods, making it difficult to isolate citrate as the reason for differences in calcium and phosphate metabolism. The concentration of citrate was lower than what was used in the present study. 


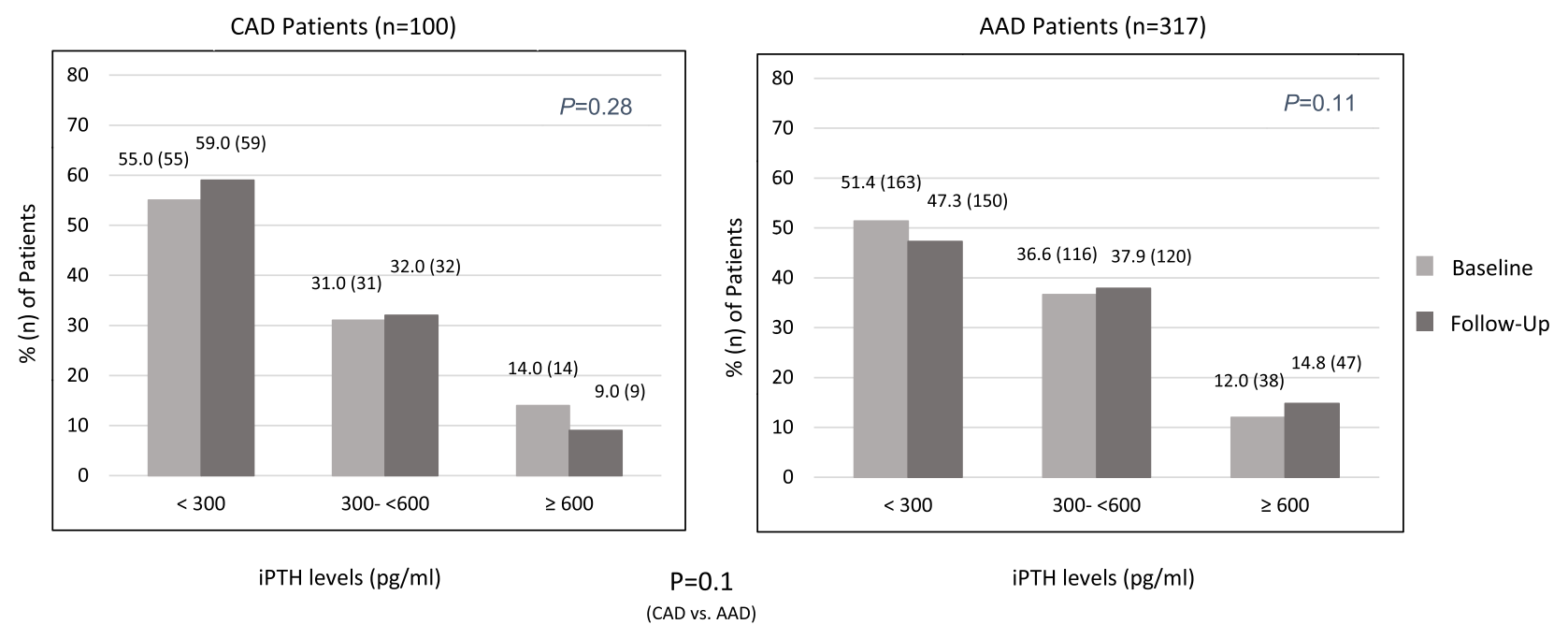

Figure 3 Individuals in each iPTH category at baseline and 6 months: patients not taking calcium-based phosphate inhibitors. Abbreviations: AAD, acetate-acidified dialysate; CAD, citrate-acidified dialysate; iPTH, intact parathyroid hormone.

Because concomitant medications could prevent a true understanding of the effects of CAD compared to AAD on serum calcium and iPTH, we evaluated the effects of CAD versus $\mathrm{AAD}$ on $\mathrm{PPTH}$ in patients who were not taking calcium-based phosphate inhibitors and patients who were not taking cinacalcet. Patients in CAD clinics did not experience a significant increase in $\mathrm{PTH}$ versus those in AAD clinics, suggesting that the impact of CAD on iPTH is minimal, regardless of additional medications, and similar to that of AAD. Similarly, the observed absence of an effect of CAD on iPTH was also observed in those patients maintaining constant IV vitamin D usage.

Considering that citrate is an effective chelator of ionized calcium, it has been suggested that hemodialysis with CAD may decrease serum-ionized calcium levels, thereby increasing PTH levels. ${ }^{23}$ Because calcium and PTH levels serve, in part, to regulate one another, ${ }^{24}$ it is also not entirely surprising that studies have indicated that pre-dialysis PTH values are modified by the existing serum calcium concentration. ${ }^{25,26}$ In our study, serum calcium changed minimally from baseline to follow-up in AAD clinics and did not change in CAD clinics. When we restricted our analysis to patients prescribed $2.5 \mathrm{mEq} / \mathrm{L}$ of calcium in the dialysate at baseline (i.e., before any potential switch to $\mathrm{CAD}$ ) and follow-up, patients in CAD clinics demonstrated significant reductions in calcium, while those remaining on AAD did not. In contrast, patients in AAD and CAD sites had similar increases in iPTH. Patients switched from dialysis with AAD containing $2.2 \mathrm{mEq} / \mathrm{L}$ of calcium at baseline to $\mathrm{CAD}$ (i.e., a 0.3
$\mathrm{mEq} / \mathrm{L}$ increase in prescribed calcium) exhibited no significant change in iPTH or serum calcium levels. Interestingly, Borrego et al have shown that in patients on hemodialysis, the PTH-calcium curve adapts to sustained changes in serum calcium levels. ${ }^{26}$ In patients with mild secondary hyperparathyroidism, changes in the set point of calcium were not correlated with changes in PTH, suggesting that the parathyroid gland can adapt to sustained changes in serum calcium.

One open-label crossover trial ( 6 weeks of AAD +6 weeks of $\mathrm{CAD} ; \mathrm{n}=24$ ) found that although post-dialysis calcium concentrations were reduced and post-dialysis PTH concentrations were increased with citrate dialysate ( $3 \mathrm{mEq} / \mathrm{L}$ citrate, $3 \mathrm{mEq} / \mathrm{L}$ calcium), the absolute concentrations were still within normal ranges, and no identified episodes of hypocalcemia occurred. ${ }^{18}$ While these results indicate that the dialysate used in the study is unlikely to result in physiologically meaningful intradialytic changes in calcium and PTH levels, it should be noted that a large longitudinal study of 53,560 hemodialysis patients from the Japan Renal Data registry found that higher dialysate calcium concentrations $(\geq 3.0 \mathrm{mEq} /$ L) were associated with greater risk of incident myocardial infarction among diabetic patients with low bone turnover. ${ }^{27}$ As such, the authors recommended avoiding dialysate calcium concentrations $\geq 3.0 \mathrm{mEq} / \mathrm{L}$ in diabetic patients with low serum calcium or iPTH levels. In contrast, a crossover, prospective study of 21 hemodialysis patients found that dialysis with citrate dialysate (containing $3.0 \mathrm{mEq} / \mathrm{L}$ of calcium) resulted in stable 
ionized calcium levels, a neutral-to-negative effect on calcium balance, and reductions in inflammatory biomarkers relative to those effects observed with acetate dialysate (containing $3.0 \mathrm{mEq} / \mathrm{L}$ of calcium). ${ }^{28}$

In the present study, both CAD and AAD clinics demonstrated a significant increase in the use of calciumbased phosphate binders from baseline to follow-up, with a greater increase in $\mathrm{AAD}$ clinics (CAD: $+7 \%$ versus AAD: $+19 \%, p=0.01)$. The increased use of calciumbased phosphate binders was not associated with an increase in serum calcium from baseline to follow-up in the CAD group, and although there was an increase in serum calcium in the AAD group, it is unclear whether calcium-based phosphate binders played a role. No significant differences were observed in changes of IV vitamin $\mathrm{D}$ over time between $\mathrm{CAD}$ and $\mathrm{AAD}$ clinics.

Patients in both CAD and AAD clinics experienced a mean increase in dialysis adequacy from baseline to follow-up, but between-clinic differences were not statistically significant. Similarly, between-group differences in pre-hemodialysis body weight and prescribed hemodialysis time were not significant. It is unlikely that minimal increases in prescribed hemodialysis time ( 1 minute, $p=$ 0.10 ) and a mean decrease in pre-dialysis body weight $(0.50 \mathrm{~kg}, p=0.03)$ accounted for the observed changes in single pool Kt/V. A small study by Gabutti et al concluded that $\mathrm{CAD}$ results in a non-significant trend toward improved $\mathrm{Kt} / \mathrm{V} .{ }^{14}$ Kossmann et al demonstrated that the use of CAD was associated with increased solute removal as measured by equilibrated $\mathrm{Kt} / \mathrm{V} .^{29}$ However, an earlier study by Ahmad et al showed that $\mathrm{Kt} / \mathrm{V}$ increased over multiple dialysis sessions with $\mathrm{CAD} .^{30}$ These effects appear to be greatest when dialyzers are reused. ${ }^{31}$

In addition to phosphate binders, vitamin $\mathrm{D}$ analogs, and calcimimetics, dialysate calcium concentration has been suggested as part of the integrated therapeutic regimen to maintain normal mineral metabolism. ${ }^{32}$ In our study, prescribed dialysate calcium increased slightly in CAD clinics from baseline to follow-up, but not in AAD clinics. This small increase could potentially be due to the tendency of clinicians to increase prescribed dialysate calcium upon switching to CAD. ${ }^{32}$ When $\mathrm{CAD}$ was introduced in France, the average dialysate calcium increased to a larger extent than that observed in our study, and it has been suggested that this increase potentially helped to reduce changes in bone mineral metabolism markers, including $\mathrm{iPTH}^{33}$
Basile et al conducted a randomized crossover trial in which 22 patients underwent three 4-hour bicarbonate hemodialysis sessions. ${ }^{32}$ Three dialysate total calcium concentrations $(2.5,2.75$, and $3.0 \mathrm{mEq} / \mathrm{L})$ were used. Investigators found that PTH levels increased during dialysis using a dialysate total calcium concentration of 2.5 $\mathrm{mEq} / \mathrm{L}$ and decreased with higher dialysate calcium concentrations of 2.75 and $3.0 \mathrm{mEq} / \mathrm{L}$. In our study, the mean prescribed dialysate calcium concentration was 2.3 to 2.5 $\mathrm{mEq} / \mathrm{L}$ at baseline and follow-up for both clinic types. We did not observe a significant increase in pre-dialysis $\mathrm{iPTH}$ from baseline to follow-up, suggesting that intra-dialytic changes in iPTH reported by Basile et al are likely transient.

Although our study did not examine patient outcomes, available evidence suggests that CAD can improve clinical outcomes. A post-marketing study from the national French Renal Epidemiology and Information Network Registry demonstrated that patients with long-term exposure to CAD (2.4-3.6 mEq/L citrate) experienced lower mortality rates than those with exposure to AAD; the crude mortality rate per 1000 patient-months was 11.5 (95\% CI: 11.1-12.0) with CAD and 12.9 (95\% CI: 12.8 13.1) with AAD. ${ }^{1}$ However, other studies have not found statistically significant differences in mortality between chronic hemodialysis patients dialyzed with CAD and AAD. A multicenter, historical cohort propensity scorematched study of adult incident hemodialysis patients found no statistically significant difference in mortality between patients dialyzed with $\mathrm{CAD}$ and patients dialyzed with AAD. ${ }^{13}$ A recent retrospective observational study conducted in France included a 5-year follow-up period and demonstrated that mortality risk is not increased with the use of CAD (relative to dialysate without citrate). ${ }^{34}$ In a recent, multicenter, randomized crossover study, CAD with $3 \mathrm{mEq} / \mathrm{L}$ calcium had a positive effect on the calcification propensity during dialysis treatment relative to conventional AAD solutions containing 3 and $2.5 \mathrm{mEq} / \mathrm{L}$ calcium. $^{3}$

Our study had several limitations. First, this is a retrospective, database analysis. We did make efforts to minimize confounding by limiting the study years and selecting matched clinics. In addition, our study did not include assessment of ionized calcium concentrations or post-dialysis laboratory parameters. Furthermore, our subgroup analysis in patients without specific medication prescriptions required a departure from the matching design of the study, and as such, the ratio of CAD:AAD clinics 
was no longer 1:4 in these analyses. Consistent findings in our subgroup analyses (i.e., excluding patients receiving cinacalcet or calcium-based phosphate binders, or with modifications of vitamin $\mathrm{D}$ usage) reduce, but do not eliminate, the impact of all confounding factors on the results. A final limitation is that our study did not evaluate patient outcomes. Future studies combining laboratory parameters with clinical outcomes could offer a more complete picture of the impact of CAD versus AAD.

Specific strengths of this study should also be noted. A contemporaneous comparison group was utilized to control for any background changes in patient management during the observation period. Matching each CAD clinic with four geographically similar AAD clinics accounted for local variations in management of these patients. Our subgroup analyses of patients who were not using calcium-based phosphate binders or cinacalcet allowed us to observe the effects of CAD and AAD without confounding effects associated with those medications.

\section{Conclusions}

Similar trends in both pre-dialysis iPTH and serum calcium levels were observed in clinics that switched from AAD to CAD and the geographically matched AAD-only clinics. Both $\mathrm{CAD}$ and $\mathrm{AAD}$ clinics showed increased dialysis adequacy, with no statistically significant difference between groups. Taken together, these results support $\mathrm{CAD}$ as a potential alternative to $\mathrm{AAD}$ in hemodialysis.

\section{Abbreviations}

AAD, acetate-acidified dialysate; CAD, citrate-acidified dialysate; CKD, chronic kidney disease; iPTH, intact parathyroid hormone; IV, intravenous; PTH, parathyroid hormone; SD, standard deviation.

\section{Data Sharing Statement}

We encourage investigators interested in data sharing and collaboration to contact the corresponding author.

\section{Ethics Approval and Consent}

This manuscript reports data from a retrospective analysis. This study was submitted to an ethics committee, New England Institutional Review Board (NEIRB; Needham, Massachusetts), and approved (NEIRB\#: 1-9452-1) under the exempt category because of use of de-identified data and purely observational nature of the study.

\section{Acknowledgments}

Medical writing and editing support was provided by NorthStar Strategic Consulting, LLC, via funding by Fresenius Medical Care Renal Therapies Group.

\section{Author Contributions}

All authors made a significant contribution to the work reported, whether that is in the conception, study design, execution, acquisition of data, analysis and interpretation, or in all these areas; took part in drafting, revising or critically reviewing the article; gave final approval of the version to be published; have agreed on the journal to which the article has been submitted; and agree to be accountable for all aspects of the work.

\section{Funding}

Fresenius Medical Care North America Renal Therapies Group provided funding for the study and is the US distributor of Citrasate. Employees of Fresenius Medical Care were involved in the design, analysis, interpretation of results, and writing. Fresenius Kidney Care is a dialysis provider, and data for the study were from the Fresenius Kidney Care clinical data warehouse.

\section{Disclosure}

LHF, MZ, CM, MSA, and RJK are employees of Fresenius Medical Care. CM and RJK own stock in Fresenius Medical Care Ag \& Co. KGaA. RJK has ownership interest in Advanced Renal Technologies and is on its board of directors. RJK is on the Scientific Advisory Board of Vifor Pharma. The authors report no other conflicts of interest in this work.

\section{References}

1. Couchoud C, Hannedouche T, Bauwens M, et al. Impact of the dialysate acid component on haemodialysis mortality rates. Nephrol Dial Transplant. 2020;35(7):1244-1249. doi:10.1093/ndt/gfaa168

2. Villa-Bellosta R, Hernández-Martínez E, Mérida-Herrero E, GonzálezParra E. Impact of acetate- or citrate-acidified bicarbonate dialysate on ex vivo aorta wall calcification. Sci Rep. 2019;9(1):1-7. doi:10.1038/ s41598-019-47934-7

3. Ter Meulen KJ, Dekker MJE, Pasch A, et al. Citric-acid dialysate improves the calcification propensity of hemodialysis patients: a multicenter prospective randomized crossover trial. PLoS One. 2019;14(12):e0225824. doi:10.1371/journal.pone.0225824

4. Calvi LM, Bushinsky DA. When is it appropriate to order an ionized calcium? J Am Soc Nephrol. 2008;19:1257-1260. doi:10.1681/ ASN.2007121327

5. Ureña-Torres PA, Vervloet M, Mazzaferro S, et al. Novel insights into parathyroid hormone: report of the parathyroid day in chronic kidney disease. Clin Kidney J. 2018;12:269-280. doi:10.1093/ckj/sfy061 
6. Brown EM, Vassilev PM, Quinn S, Hebert SC. G-protein-coupled, extracellular $\mathrm{Ca} 2+-$-sensing receptor: a versatile regulator of diverse cellular functions. Vitam Horm. 1998;55(C):1-71.

7. Marx SJ. Hyperparathyroid and hypoparathyroid disorders. $N$ Engl $J \quad$ Med. 2000;343(25):1863-1875. doi:10.1056/NEJM200 012213432508

8. Martinez I, Saracho R, Montenegro J, Llach F. The importance of dietary calcium and phosphorous in the secondary hyperparathyroidism of patients with early renal failure. Am J Kidney Dis. 1997;29 (4):496-502. doi:10.1016/S0272-6386(97)90330-9

9. Johnson CA, McCarthy J, Bailie GR, Deane J, Smith S. Analysis of renal bone disease treatment in dialysis patients. Am J Kidney Dis. 2002;39(6):1270-1277. doi:10.1053/ajkd.2002.33401

10. Lindberg JS, Culleton B, Wong $\mathrm{G}$, et al. Cinacalcet $\mathrm{HCl}$, an oral calcimimetic agent for the treatment of secondary hyperparathyroidism in hemodialysis and peritoneal dialysis: a randomized, double-blind, multicenter study. $J$ Am Soc Nephrol. 2005;16 (3):800-807. doi:10.1681/ASN.2004060512

11. Hwang E, Choi BS, Oh KH, Kwon YJ, Kim GH. Management of chronic kidney disease-mineral and bone disorder: Korean working group recommendations. Kidney Res Clin Pract. 2015;34(1):4-12. doi:10.1016/j.krcp.2015.02.002

12. Martin KJ, González EA, Gellens M, Hamm LL, Abboud H, Lindberg J. 19-Nor-1-alpha-25-dihydroxyvitamin D2 (Paricalcitol) safely and effectively reduces the levels of intact parathyroid hormone in patients on hemodialysis. J Am Soc Nephrol. 1998;9 (8):1427-1432. doi:10.1681/ASN.V981427

13. Neri L, Bellocchio F, Kircelli F, et al. Long-Term mortality risk associated with citric acid- and acetic acid-based bicarbonate haemodialysis: a historical cohort propensity score-matched study in a large, multicentre, population-based study. Nephrol Dial Transplant. 2020;35(7):1237-1244. doi:10.1093/ndt/gfaa089

14. Gabutti L, Lucchini B, Marone C, Alberio L, Burnier M. Citrate- vs. acetate-based dialysate in bicarbonate haemodialysis: consequences on haemodynamics, coagulation, acid-base status, and electrolytes. BMC Nephrol. 2009;10(1):7. doi:10.1186/1471-2369-10-7

15. Tu A, Ahmad S. Heparin-free hemodialysis with citrate-containing dialysate in intensive care patients. Dial Transplant. 2000;29 (10):620-626.

16. Kossmann RJ, Callan R, Sultan A. Fifty-five percent heparin reduction is safe with citrate dialysate in chronic dialysis patients. $J \mathrm{Am}$ Soc Nephrol. 2006;17:109-110.

17. Sands JJ, Kotanko P, Segal JH, et al. Effects of citrate acid concentrate $\left(\right.$ Citrasate $^{\mathbb{R}}$ ) on heparin $\mathrm{N}$ requirements and hemodialysis adequacy: a multicenter, prospective noninferiority trial. Blood Purif. 2012;33(1-3):199-204. doi:10.1159/000334157

18. Grundström G, Christensson A, Alquist M, Nilsson LG, Segelmark M. Replacement of acetate with citrate in dialysis fluid: a randomized clinical trial of short term safety and fluid biocompatibility. BMC Nephrol. 2013;14(1):216. doi:10.1186/14712369-14-216

19. Šafránek R, Moučka P, Vávrová J, Palička V, Pavlíková L, Sulková SD. Changes of serum calcium, magnesium and parathyroid hormone induced by hemodialysis with citrate-enriched dialysis solution. Kidney Blood Press Res. 2015;40(1):13-21. doi:10.1159/ 000368478

20. Kossmann RJ, Anderson L, Ficociello LH, Balter P, Topping A, Mullon C. Effects of Citrasate ${ }^{\circledR}$ and Citrasate Dry ${ }^{\circledR}$ on serum calcium and iPTH. ASN Kidney Week; 2019; Washington DC.
21. Park EJ, Jung SW, Kim DR, et al. Conversion from acetate dialysate to citrate dialysate in a central delivery system for maintenance hemodialysis patients. Kidney Res Clin Pract. 2019;38(1):100-107. doi:10.23876/j.krcp.18.0045

22. Ketteler M, Elder GJ, Evenepoel P, et al. Revisiting KDIGO clinical practice guideline on chronic kidney disease-mineral and bone disorder: a commentary from a kidney disease: improving global outcomes controversies conference. Kidney Int. 2015;87(3):502-508. doi:10.1038/ki.2014.425

23. Kuragano T, Furuta M, Yahiro $M$, et al. Acetate free citrate-containing dialysate increase intact-PTH and BAP levels in the patients with low intact-PTH. BMC Nephrol. 2013;14(1):18. doi:10.1186/1471-2369-14-18

24. Felsenfeld AJ, Rodríguez M, Aguilera-Tejero E. Dynamics of parathyroid hormone secretion in health and secondary hyperparathyroidism. Clin J Am Soc Nephrol. 2007;2(6):1283-1305. doi:10.2215/CJN.01520407

25. Pahl M, Jara A, Bover J, Rodriguez M, Felsenfeld AJ. The set point of calcium and the reduction of parathyroid hormone in hemodialysis patients. Kidney Int. 1996;49(1):226-231. doi:10.1038/ki.1996.31

26. Borrego MJ, Felsenfeld AJ, Martin-Malo A, et al. Evidence for adaptation of the entire PTH-calcium curve to sustained changes in the serum calcium in haemodialysis patients. Nephrol Dial Transplant. 1997;12(3):505-513. doi:10.1093/ndt/12.3.505

27. Tagawa M, Hamano T, Sueta S, Ogata S, Saito Y. Higher dialysate calcium concentration is associated with incident myocardial infarction among diabetic patients with low bone turnover: a longitudinal study. Sci Rep. 2018;8(1):10060. doi:10.1038/s41598-018-28422-w

28. Broseta JJ, López-Romero LC, Cerveró A, et al. Improvements in inflammation and calcium balance of citrate versus acetate as dialysate buffer in maintenance hemodialysis: a unicentric, cross-over, prospective study. Blood Purif. 2021;50(6):914-920. doi:10.1159/ 000513419

29. Kossmann RJ, Gonzales A, Callan R, Ahmad S. Increased efficiency of hemodialysis with citrate dialysate: a prospective controlled study. Clin J Am Soc Nephrol. 2009;4(9):1459-1464. doi:10.2215/ CJN.02590409

30. Ahmad S, Callan R, Cole J, Blagg C. Dialysate made from dry chemicals using citric acid increases dialysis dose. Am J Kidney Dis. 2000;35(3):493-499. doi:10.1016/S0272-6386(00)70203-4

31. Ahmad S, Callan R, Cole J, Blagg C. Increased dialyzer reuse with citrate dialysate. Hemodial Int. 2005;9(3):264-267. doi:10.1111/ j.1492-7535.2005.01140.x

32. Basile C, Libutti P, Di Turo AL, et al. Effect of dialysate calcium concentrations on parathyroid hormone and calcium balance during a single dialysis session using bicarbonate hemodialysis: a crossover clinical trial. Am J Kidney Dis. 2012;59(1):92-101. doi:10.1053/j. ajkd.2011.08.033

33. Potier J, Dolley-Hitze T, Hamel D, et al. Long-term effects of citric acid-based bicarbonate haemodialysis on patient outcomes: a survival propensity score-matched study in western France. Nephrol Dial Transplant. 2020;35(7):1228-1236. doi:10.1093/ndt/gfz274

34. Séret G, Durand P, El-Haggan W, et al. Impact of long-term citrate dialysate use on survival in haemodialysis patients. Blood Purif. 2020;49(6):765-766. doi:10.1159/000502939 


\section{Publish your work in this journal}

The International Journal of Nephrology and Renovascular Disease is an international, peer-reviewed open-access journal focusing on the pathophysiology of the kidney and vascular supply. Epidemiology, screening, diagnosis, and treatment interventions are covered as well as basic

science, biochemical and immunological studies. The manuscript management system is completely online and includes a very quick and fair peer-review system, which is all easy to use. Visit http://www.dovepress.com/testimonials.php to read real quotes from published authors.

Submit your manuscript here: https://www.dovepress.com/international-journal-of-nephrology-and-renovascular-disease-journal 